\title{
Characterization of Structural Change of Nano-crystalline ITO Films due to Exposure to Hyperthermal Atomic Oxygen
}

\author{
Long Li*, Ross Harder**, Fengting Xu*, Ian K. Robinson** and Judith C. Yang* \\ *Department of Materials Science and Engineering, University of Pittsburgh, Pittsburgh, PA 15261. \\ **Department of Physics, University of Illinois at Urbana-Champaign, Urbana, IL 61801, USA.
}

The primary species in the low Earth Orbit (LEO, latitude from 180 to $650 \mathrm{~km}$ ) are vacuum ultraviolet (VUV) radiation, atomic oxygen, X-rays, electrons and other charged species. The atomic oxygen $(\mathrm{AO})$ is produced from the $\mathrm{O}_{2}$ photodissociation by solar ultraviolet. The highly reactive hyperthermal $\mathrm{AO}$ with a $5 \mathrm{eV}$ kinetic energy can cause rapid oxidation and degradation of the materials in the LEO. Indium-tin-oxide (ITO), known as a transparent conductive oxide, has been typically used as a coating material to protect Kapton blankets of solar arrays from AO-erosion and to bleed off static charge from the insulating polymeric surface [1, 2]. An AO source which produces $5 \mathrm{eV}$ hyperthermal atomic oxygen from breakdown of $\mathrm{O}_{2}$ by laser detonation, was employed to expose ITO films grown on float glass to investigate the reaction mechanism of ITO with atomic oxygen. The samples were characterized before and after $\mathrm{AO}$ exposure at room temperature with scanning electron microscope (SEM), atomic force microscope (AFM), synchrotron X-ray diffraction (XRD), and cross-sectional (scanning) transmission electron microscope ((S)TEM).

Fig. 1 is a set of images of structural characterizations of the ITO films before AO exposure. The SEM image (1a) shows that ITO grains on the surface distributed in bundles in two types of shapes, one with round shape in diameters ranging 20-50 nm and the other one with needle-like shape of 2050 in width by $\sim 100 \mathrm{~nm}$ in length. The AFM image (1b) shows the ITO surface with an RMS roughness of $5.3 \mathrm{~nm}$. Fig. 1c is a cross-sectional TEM image which shows the columnar growth of grains in the ITO film with a $200 \mathrm{~nm}$ thickness. The inset selective area electron diffraction pattern (SAD) revealed that the ITO film is polycrystalline. A layer of $\sim 40 \mathrm{~nm}$ amorphous $\mathrm{SiO}_{2}$ was observed on the substrate. The interface of $\mathrm{ITO} / \mathrm{SiO}_{2}$ is abrupt and clean without interfacial phase. Fig. 1d is an HRTEM image of a typical grain boundary. The broad transition area at the boundary indicates the non-contacting boundary of the finger like grains. Cross-sectional TEM samples were made from the ITO films after exposed to AO with a fluence of $2 \times 10^{20}$ atom $/ \mathrm{cm}^{2}$ and the results are shown in Fig. 2 . Many spherical particles (sizes: 2 to $10 \mathrm{~nm}$, Fig. 2a) appeared in the $\mathrm{SiO}_{2}$ substrate near interface, as compared to the clean interface of the unexposed ITO film (Fig. 1c). HRTEM image on Fig. 2b shows that these particles are well-crystallized, whose lattice parameters fit to that of the ITO JCPDS file. The particles were characterized with EDS, as shown in Fig. $2 \mathrm{~d}$ with a reference HAADF image (Fig. 2c). The EDS detections revealed the particles were ITO particles immersed in the $\mathrm{SiO}_{2}$ substrate. This is consistent with our synchrotron XRD results (not shown) that the crystallinity in the ITO film and at the interface was significantly modified due to AO exposure. This may be detrimental to the application of ITO coating to protect polymers from AO erosion in LEO.

\section{References}

[1] A. E. Hedin, Journal of Geophysical Research, [Space Physics] 92 (1987) 4649-4662.

[2] L. Li et al., Mat. Res. Soc. Symp. Proc. (2006) Q 10.8.

[3] This Multi-University Research Initiative (MURI) is supported through the AFOSR (F49620-011-0336). Some characterizations were performed in the Center for Microanalysis of Materials, at University of Illinois at Urbana-Champaign, which is partially supported by the U.S. DoE (DEFG02- 
96-ER45439). Authors thank L. Wang, J.G. Wen, A.M. Stewart and C.Van Ormer for their help.

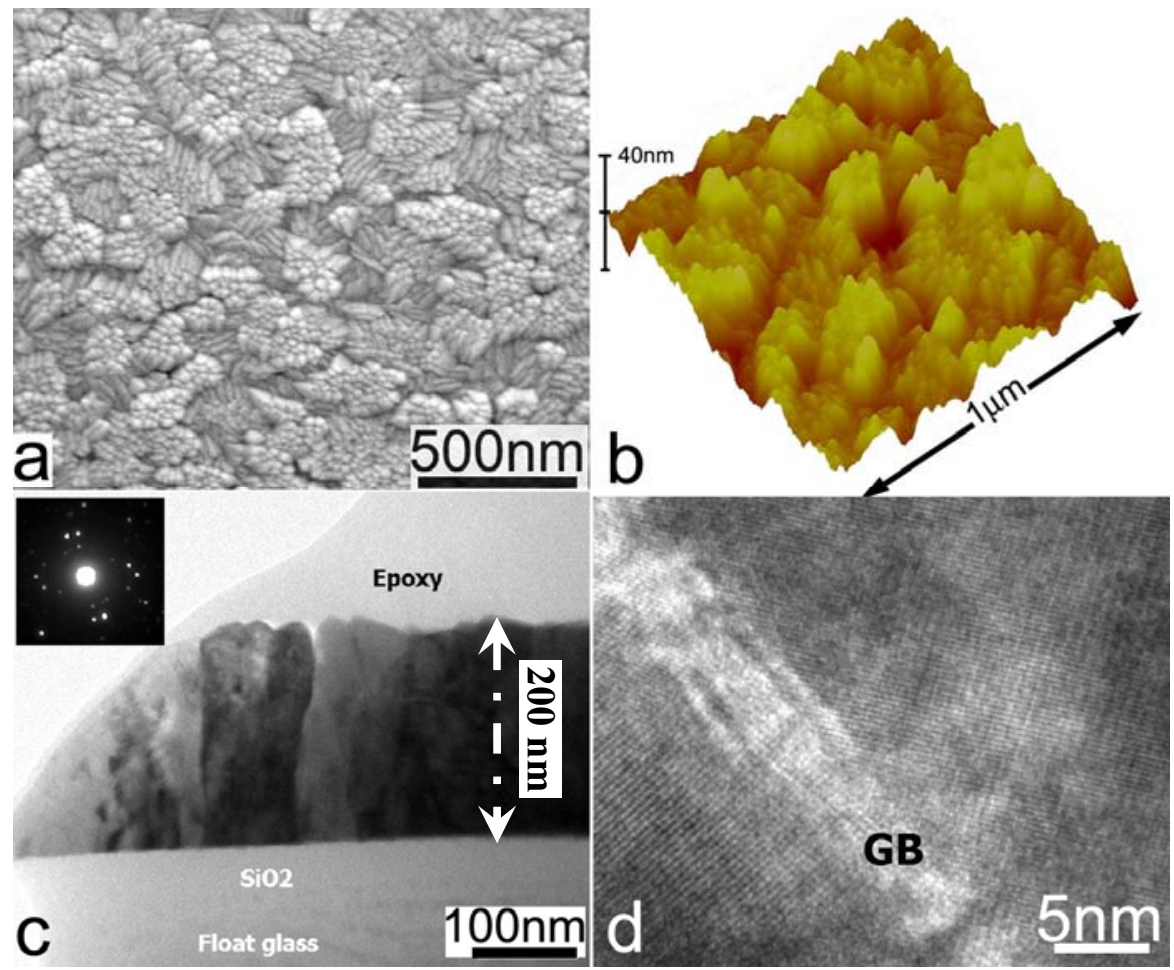

Fig. 1. Structural characterizations for ITO films before $\mathrm{AO}$ exposure. Fig. $1 \mathrm{a}$ and $1 \mathrm{~b}$ are SEM and AFM images of ITO film surface; Fig. 1c is a cross-sectional TEM image and inset SAD pattern; Fig. $1 \mathrm{~d}$ is an HRTEM image, showing a grain boundary of two columnar grains.
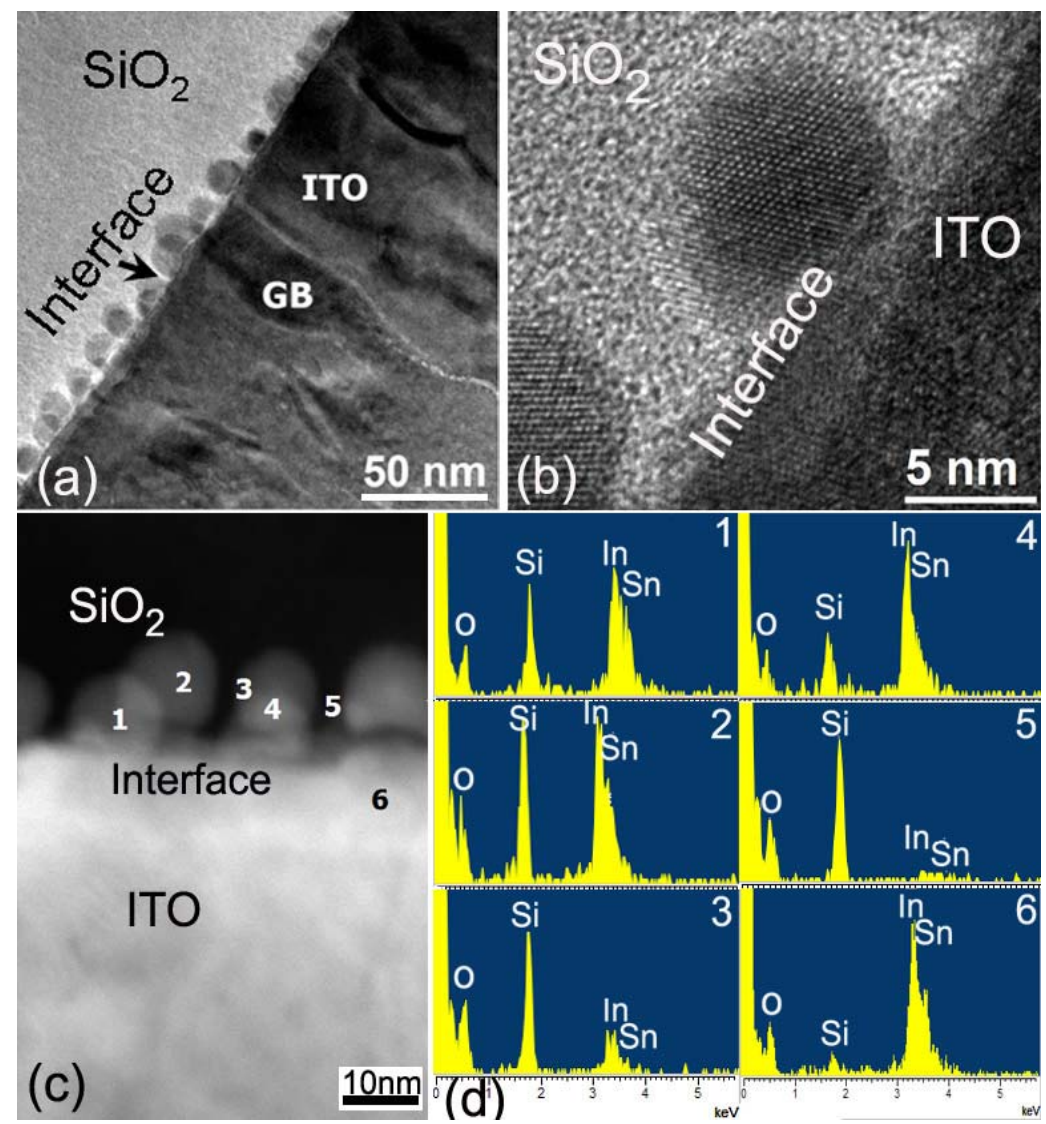

Fig. 2. Cross-sectional (S)TEM investigations of ITO films after exposed to $\mathrm{AO}$ with a fluence of $2 \times 10^{20}$ atom $/ \mathrm{cm}^{2}$ at room temperature. Fig. 2a is a Cross- sectional TEM image at $\mathrm{ITO} / \mathrm{SiO}_{2}$ interface; Fig. 2b is an HRTEM at ITO/ $/ \mathrm{SiO}_{2}$ interface; Fig. 2c is a HAADF image; and Fig. $2 \mathrm{~d}$ is a set of EDS spectra acquired from the position 1 through 6 as shown in Fig. 2c. 